Pacific Journal of Mathematic

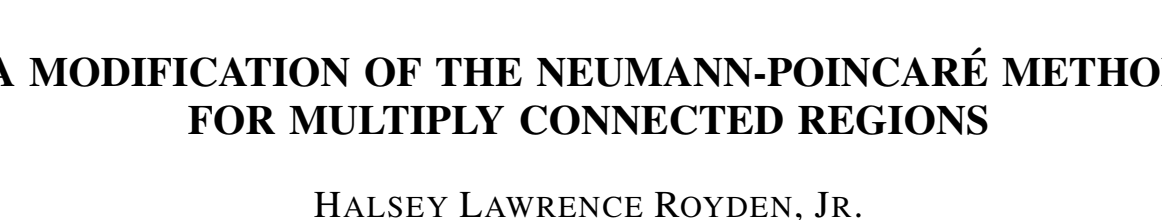




\title{
A MODIFICATION OF THE NEUMANN-POINCARÉ METHOD FOR MULTIPLY CONNECTED REGIONS
}

\author{
H. L. ROYDEN
}

1. Introduction. Some interest has been attached to the problem of effective computation of the solution to boundary value and conformal mapping problems. Birkhoff $[3 ; 4]$ has given an excellent iteration precedure for the solution of the conformal mapping problem for simply connected regions. However, the convergence of his method is easily seen to be the same as that for the classical Neumann-Poincaré method in potential theory, which, while converging for all simply connected regions $[1 ; 5]$, fails to converge for the computation of the harmonic measures of multiply connected regions $[1 ; 2]$. Since it is primarily these harmonic measures which are needed in the conformal mapping problem, we derive in the present paper a modification of the Neumann-Poincaré method which will converge in this case and apply it to the conformal mapping of doubly connected regions. While the formulas involve certain $\vartheta$-series, these should not present a major problem for numerical computation since the series are very rapidly convergent and only a few terms need be taken.

2. General formulation. Let $\Omega$ be a multiply connected region which is bounded by a smooth curve $C$. Let $U(z)$ be a continuous real-valued function defined for $z \in C$. We wish to consider the problem of finding a function $u+i v$ which is analytic in $\Omega$ and for which

$$
u(\zeta) \rightarrow U(z), \quad \text { as } \zeta \rightarrow z \in C .
$$

We do not require that $v$ be single valued in $\Omega$, and we shall assume that at a fixed point $\zeta_{0} \in C$ we have

$$
U\left(\zeta_{0}\right)=0
$$

Let $W$ be a Riemann surface which contains $\Omega$ and for which $\tilde{\Omega}=W-\Omega$ is also a connected region. For example, if $\Omega$ is the plane region exterior to the contours $C_{1}, \ldots, C_{n-1}$ and interior to $C_{n}$, we may take $W$ to be the double of the region $R$ which is bounded by circles $\gamma_{1}, \cdots, \gamma_{n}$, where $\gamma_{i}$ lies inside

Received September 12, 1951. Work done under contract N5ori-07634, between the Office of Naval Research and Harvard University.

Pacific J. Math 2 (1952), 385-394 
$C_{i}$ for $i=1, \cdots, n-1$, and $\gamma_{n}$ lies outside $C_{n}$.

If $\mathrm{g}\left(z ; \zeta, \zeta_{0}\right)$ is the Green's function for $W$, that is, a harmonic function of the variable $z$ which has a positive logarithmic pole at $\zeta$ and a negative logarithmic pole at $\zeta_{0}$, we have the symmetry relation

$$
g\left(z ; \zeta, \zeta_{0}\right)-g\left(z_{0} ; \zeta, \zeta_{0}\right)=g\left(\zeta ; z, z_{0}\right)-g\left(\zeta_{0} ; z, z_{0}\right) .
$$

From this it follows that the derivatives of $g$ with respect to $x$ and $y$ (where $z=x+i y)$ are harmonic functions of $\zeta$.

Thus if $\mu(z)$ is a continuous real-valued function defined for $z \in C$, then the function

$$
u_{i}(\zeta)=\frac{1}{2 \pi} \int_{C} \mu(z) \frac{\partial g}{\partial n_{z}} d s_{z}
$$

(where $n$ is the inner normal to $\Omega$ ) is a harmonic function of $\zeta$. If further we define

$$
u_{e}(\zeta)=\frac{1}{2 \pi} \int_{C} \mu(z) \frac{\partial g}{\partial n_{z}} d s_{z}
$$

then we know by the well-known boundary behavior of double layer distributions that

$$
\frac{\partial u_{e}(\zeta)}{\partial n}=\frac{\partial u_{i}(\zeta)}{\partial n}
$$

while

$$
u_{i}(\zeta)=-\frac{1}{2} \mu(\zeta)+\frac{1}{2} \mu\left(\zeta_{0}\right)+\frac{1}{2 \pi} \int_{C} \mu(z) \frac{\partial g}{\partial n_{z}} d s_{z}
$$

and

$$
u_{e}(\zeta)=\frac{1}{2} \mu(\zeta)-\frac{1}{2} \mu\left(\zeta_{0}\right)+\frac{1}{2 \pi} \int_{C} \mu(z) \frac{\partial g}{\partial n_{z}} d s_{z}
$$

If we suppose that $\mu(\zeta)=0$, then the solution of our problem is determined if we can solve the integral equation

$$
2 U(\zeta)=-\mu(\zeta)+\frac{1}{\pi} \int_{C} \mu(z) \frac{\partial g}{\partial n_{z}} d s_{z},
$$

for $\mu$ subject to the condition that $\mu\left(\zeta_{0}\right)=0$. If this is done, then $u$ is given by 
(7), and $v$ is given by

$$
v=\frac{1}{2 \pi} \int_{C} \mu(z) \frac{\partial h}{\partial n_{z}} d s_{z},
$$

where $\partial h / \partial n_{z}\left(z ; \zeta, \zeta_{0}\right)$ is the harmonic conjugate with respect to $\zeta$ of $\partial g / \partial n_{z} \quad(z$; $\left.\zeta, \zeta_{0}\right)$; that is, such that

$$
\frac{\partial g}{\partial n}+i \frac{\partial h}{\partial n}
$$

is an analytic function of $\zeta$.

3. Solution of the integral equation. Because of the smoothness of $C$, we know that $\partial g / \partial n_{z}$ is a continuous function on $C$, and the Fredholm theory states that either

$$
2 U(\zeta)=-\mu(\zeta)+\frac{\lambda}{\pi} \int_{C} \mu(z) \frac{\partial g}{\partial n_{z}} d s_{z}
$$

has a unique continuous solution for all continuous functions $U(z)$, or else

$$
\mu(\zeta)=\frac{\lambda}{\pi} \int_{C} \mu(z) \frac{\partial g}{\partial n_{z}} d s_{z}
$$

has a nontrivial solution. Moreover, if we set

$$
T[\phi]=\frac{1}{\pi} \int_{C} \phi \frac{\partial g}{\partial n_{z}} d s_{z},
$$

we have

$$
\mu(z)=-2 \sum_{\nu=0}^{\infty} \lambda^{\nu} T^{\nu} U,
$$

uniformly in $z$ for all $\lambda$ with $|\lambda|<\left|\lambda_{0}\right|$, where $\lambda_{0}$ is the absolutely smallest value of $\lambda$ for which (12) has a nontrivial solution. Thus if we can show that $\left|\lambda_{0}\right| \geq M>1$, we know that the series

$$
\mu(z)=-2 \sum_{\nu=0}^{\infty} T^{\nu} U
$$

converges with order at least $1 / M$ and gives a solution to the integral equation (9). For computational purposes, the series (15) is more conveniently expressed 
by the following iteration:

$$
\begin{aligned}
& \mu_{1}(\zeta)=-2 U(\zeta) \\
& \mu_{n}(\zeta)=-2 U(\zeta)+T \mu_{n-1} \cdot
\end{aligned}
$$

In order to ensure the validity of this iterative method, we need only to bound the eigenvalues of (12) away from unity. Suppose that we have an eigenfunction

$$
\phi=\lambda_{1} T \phi \text {. }
$$

Then

$$
u_{i}(\zeta)=\frac{1}{2 \pi} \int_{C} \phi(z) \frac{\partial g}{\partial n_{z}} d s_{z}
$$

and

$$
u_{e}(\zeta)=\frac{1}{2 \pi} \int_{C} \phi(z) \frac{\partial g}{\partial n_{z}} d s_{z}
$$

are harmonic functions in $\Omega$ and $\tilde{\Omega}$, respectively. For $\zeta \in C$ we have, by (7) and (8),

$$
u_{i}(\zeta)=-\frac{1}{2} \phi(\zeta)+\frac{1}{2 \pi} \int_{C} \phi(z) \frac{\partial g}{\partial n} d s=-\frac{1}{2}\left(1-\frac{1}{\lambda_{1}}\right) \phi(\zeta) .
$$

Also,

$$
u_{e}=+\frac{1}{2} \phi(\zeta)+\frac{1}{2 \pi} \int_{C} \phi(z) \frac{\partial g}{\partial n} d s=\frac{1}{2}\left(1+\frac{1}{\lambda_{1}}\right) \phi(\zeta),
$$

whence

$$
u_{i}(\zeta)=\frac{1-\lambda_{1}}{1+\lambda_{1}} u_{e}(\zeta)
$$

By (6) we have

$$
\frac{\partial u_{i}}{\partial n}=\frac{\partial u_{e}}{\partial n}
$$

and so

$$
D\left(u_{i}\right)=\int_{C} u_{i} \frac{\partial \bar{u}_{i}}{\partial n} d s=\frac{1-\lambda_{1}}{1+\lambda_{1}} \int_{C} u_{e} \frac{\partial \bar{u}_{e}}{\partial n} d s=\frac{\lambda_{1}-1}{\lambda_{1}+1} D\left(u_{e}\right),
$$


where

$$
D\left(u_{i}\right)=\iint_{\Omega}\left\{\left|\frac{\partial u_{i}}{\partial x}\right|^{2}+\left|\frac{\partial u_{i}}{\partial y}\right|^{2}\right\} d x d y
$$

and

$$
D\left(e_{u}\right)=\iint_{\tilde{\Omega}}\left\{\left|\frac{\partial u_{e}}{\partial x}\right|^{2}+\left|\frac{\partial u_{e}}{\partial y}\right|^{2}\right\} d x d y .
$$

Thus

$$
\lambda_{1}=\frac{r+1}{r-1}
$$

where

$$
r=\frac{D\left(u_{e}\right)}{D\left(u_{i}\right)}
$$

Since $D\left(u_{e}\right)$ and $D\left(u_{i}\right)$ are always nonnegative, we conclude that $\lambda_{1}$ is real and

$$
\left|\lambda_{1}\right| \geq 1
$$

In order for equality to occur in (19), we must have either $u_{e}$ constant or $u_{i}$ constant. In either case the constant must be zero since $u_{i}\left(\zeta_{0}\right)=0$ and $u_{e}\left(\zeta_{0}\right)=$ 0 , while $\Omega$ and $\tilde{\Omega}$ are connected. Thus equality is excluded in (19) and we have, taking $\lambda_{1}$ to be $\lambda_{0}$, the absolutely smallest eigenvalue,

$$
\left|\lambda_{0}\right|>1
$$

this ensures the convergence and correctness of the iteration (16).

In order to obtain an estimate for $\left|\lambda_{0}\right|$ and consequently for the rate of convergence of (20), we suppose that $\Omega$ and $\tilde{\Omega}$ are topologically equivalent. Then following Ahlfors [1] we take a quasi-conformal mapping $f(z)$ of $\Omega$ onto $\tilde{\Omega}$ with dilation quotient $\leq K$. Then the functions $v_{i}$ and $v_{e}$, which are the harmonic conjugates of $u_{i}$ and $u_{e}$ with the conditions that $v_{i}\left(\zeta_{0}\right)=v_{e}\left(\zeta_{0}\right)=0$, are harmonic functions in $\Omega$ and $\tilde{\Omega}$ with the same values on $C$ by (6). Moreover, $D\left(v_{i}\right)=$ $D\left(u_{i}\right) ; D\left(v_{e}\right)=D\left(u_{e}\right)$.

But now $v_{e}[f(z)]$ is a continuous function in $\Omega$ which has the same periods and boundary values as $v_{i}$. Hence by the Dirichlet principle we have

$$
D\left\{v_{e}[f(z)]\right\} \geq D\left\{v_{i}\right\}
$$


But

$$
D\left\{v_{\boldsymbol{e}}[f(z)]\right\} \leq K D\left\{v_{\boldsymbol{e}}\right\}
$$

Hence

$$
K \geq \frac{D\left\{v_{i}\right\}}{D\left\{v_{e}\right\}}=\frac{\lambda_{0}-1}{\lambda_{0}+1} .
$$

In a similar manner, using $f^{-1}$, we obtain

$$
K \geq \frac{D\left\{v_{e}\right\}}{D\left\{v_{i}\right\}}=\frac{\lambda_{0}+1}{\lambda_{0}-1} \text {. }
$$

Hence either

$$
\lambda_{0} \leq-\frac{(K+1)}{(K+1)} \text {, or } \lambda_{0} \geq \frac{K+1}{K-1}
$$

that is,

$$
\left|\lambda_{0}\right| \geq \frac{K+1}{K-1}
$$

4. The doubly connected case. We now suppose that $\Omega$ is a doubly connected region in the plane contained in the circle $|z| \leq 1$ and containing the circle $|z| \leq q^{2}$ within its inner contour. We take our Riemann surface $W$ to be the torus formed by identifying the points $z$ and $q^{2} z$.

Now we shall show that the Green's function for $W$ is

$$
g\left(z ; \zeta, \zeta_{0}\right)=\log \left|\frac{\vartheta_{*}\left(q z / \zeta_{0}\right)}{\vartheta_{*}(q z / \zeta)}\right|-\frac{1}{2} \frac{\log |z|}{\log q} \cdot \log \left|\frac{\zeta_{0}}{\zeta}\right|,
$$

where

$$
\vartheta_{*}(t)=\vartheta_{4}\left(\frac{1}{2 i} \log t, q\right)=1+\sum_{n=1}^{\infty}(-)^{n} q^{n^{2}}\left(t^{n}+t^{-n}\right)
$$

From (23), we see that $\vartheta_{*}$ is a single-valued function of $t$ and that

$$
\begin{aligned}
\vartheta_{*}\left(q^{2} t\right)=\sum_{n=-\infty}^{\infty}(-)^{n} q^{n^{2}+2 n} t^{n} & =-(q t)^{-1} \sum_{n=-\infty}^{\infty}(-)^{n+1} q^{(n+1)} t^{n+1} \\
& =-(q t)^{-1} \vartheta_{*}(t) .
\end{aligned}
$$


Hence

$$
\begin{aligned}
g\left(q^{2} z ; \zeta, \zeta_{0}\right) & =\log \frac{\left|\vartheta_{*}\left(q z / \zeta_{0}\right)\right|\left|\zeta_{0}\right|}{\left|\vartheta_{*}(q z / \zeta)\right||\zeta|}-\frac{1}{2} \frac{\log |z|}{\log q} \log \left|\frac{\zeta_{0}}{\zeta}\right|-\log \left|\frac{\zeta_{0}}{\zeta}\right| \\
& =g\left(z ; \zeta, \zeta_{0}\right),
\end{aligned}
$$

so that $g$ is a single-valued function of $z$ on the torus. By (23) we see that $g$ has poles at $\zeta$ and $\zeta_{0}$, since

$$
\vartheta_{*}(q)=0 \text {. }
$$

Moreover, $\vartheta_{4}$ has only one zero for $(1 / 2 i) \log t$ in the rectangle whose corners are $a, a+\pi, a+\pi-i \log q, a-i \log q\left[6\right.$, p. 465]. Thus $\vartheta_{*}(t)$ has only one zero for $q^{2} \leq|t| \leq 1$, and consequently $g$ is the Green's function of $W$.

5. Computation. The iteration (16) becomes now

$$
\begin{aligned}
& \mu_{1}(\zeta)=-2 U(\zeta), \\
& \mu_{n}(\zeta)=-2 U(\zeta)-\frac{2}{\pi} \int_{C} \mu_{n-1}(z) d \frac{\partial g}{\partial z} z^{\prime}(s) d s,
\end{aligned}
$$

since

$$
\frac{\partial g}{\partial n}=\frac{\partial g}{\partial z} \frac{\partial z}{\partial n}+\frac{\partial g}{\partial \bar{z}} \frac{\partial \bar{z}}{\partial n}=2 R \frac{\partial g}{\partial z} \frac{\partial z}{\partial n}
$$

while

$$
\frac{\partial z}{\partial n}=i \frac{\partial z}{\partial s}
$$

and so

$$
\begin{aligned}
\frac{\partial g}{\partial n} & =2 R\left\{i \frac{\partial g}{\partial z} \frac{\partial z}{\partial s}\right\} \\
& =-2 d \frac{\partial g}{\partial z} z^{\prime} .
\end{aligned}
$$

Clearly (24) is independent of the choice of the parameter $s$, and hence $s$ need not be arc-length, but may be any conveniently chosen parameter $\sigma$.

Now

$$
g\left(z ; \zeta, \zeta_{0}\right)=\log \left|\frac{\vartheta_{*}\left(q z / \zeta_{0}\right)}{\vartheta_{*}(q z / \zeta)}\right|=\frac{1}{2} \frac{\log |z|}{\log q} \log \left|\frac{\zeta_{0}}{\zeta}\right|,
$$


whence

$$
2 \frac{\partial g}{\partial z}=f(z, \zeta)-f\left(z, \zeta_{0}\right)
$$

where

$$
f(z, \zeta)=\frac{-\vartheta_{*}^{\prime}(q z / \zeta) q / \zeta}{\vartheta_{*}(q z / \zeta)}+\frac{1}{2 z} \cdot \frac{\log |\zeta|}{\log q}
$$

Thus (24) becomes

$$
\begin{aligned}
& \mu_{1}(\zeta)=-2 U(\zeta) \\
& \mu_{n}(\zeta)=-2 U(\zeta)-\frac{1}{\pi} \int_{C} \mu_{n-1}(z) d\left[f(z, \zeta)-f\left(z, \zeta_{0}\right)\right] z^{\prime} d s .
\end{aligned}
$$

This is equivalent to

$$
\begin{aligned}
& -\mu_{n}^{(0)}=-2 U(\zeta)-\frac{1}{\pi} \int_{C} \mu_{n-1}(z) d f(z, \zeta) z^{\prime} d s \\
& \mu_{n}(\zeta)=\mu_{n}^{(0)}(\zeta)-\mu_{n}^{(0)}(\zeta) .
\end{aligned}
$$

Near $z=\zeta$ the boundary has the following expansion in terms of arc length $s:$

$$
z=\zeta+\zeta^{\prime}\left[s+\frac{1}{2} i \kappa s^{2}+O\left(s^{3}\right)\right]
$$

where primes denote differentiation with respect to arc-length and $\kappa$ is the curvature at $\zeta$. Then, since

$$
\frac{\vartheta_{*}^{\prime \prime}(q)}{\vartheta_{*}^{\prime}(q)}=-\frac{2}{q}
$$

we have

$$
\vartheta_{*}\left(\frac{q z}{\zeta}\right)=\vartheta_{*}^{\prime}(q)\left[q \frac{\zeta^{\prime}}{\zeta}\left(s+\frac{1}{2} i \kappa s^{2}\right)-\frac{1 q^{2} \zeta^{2}}{q \zeta^{2}} s^{2}+O\left(s^{3}\right)\right] .
$$

Also

$$
\vartheta_{*}^{\prime}\left(\frac{q z}{\zeta}\right)=\vartheta_{*}^{\prime}(q)\left[1-\frac{2 \zeta^{\prime}}{\zeta} s+O\left(s^{2}\right)\right],
$$

while 


$$
\frac{q z^{\prime}}{\zeta}=\frac{q \zeta^{\prime}}{\zeta}\left[1+i \kappa s+O\left(s^{2}\right)\right]
$$

Hence

$$
\frac{\vartheta_{*}^{\prime}(q z / \zeta) q z^{\prime} / \zeta}{\vartheta_{*}(q z / \zeta)}=\frac{1}{s}\left[1+\frac{1}{2} i \kappa s-\frac{\zeta^{\prime}}{\zeta} s+O\left(s^{2}\right)\right]
$$

whence

$$
d f(z, z) z^{\prime}=\frac{1}{2} \kappa(z)-d \frac{z^{\prime}}{z}\left(1-\frac{1}{2} \frac{\log |z|}{\log q}\right) .
$$

In terms of an arbitrary parameter $\sigma$, this becomes

$$
\ell f(z, z) z^{\prime}=\frac{1}{2} \kappa(z) \frac{d s}{d \sigma}-d \frac{z^{\prime}}{z}\left(1-\frac{1}{2} \frac{\log |z|}{\log q}\right),
$$

where now the primes denote differentiation with respect to $\sigma$.

For computational purposes, it would seem best to calculate

$$
\vartheta_{*}(t)=1+\sum_{n=1}^{\infty}(-)^{n} q^{n^{2}}\left(t^{n}+t^{-n}\right)
$$

and

$$
t \vartheta_{*}^{\prime}(t)=\sum_{n=1}^{\infty}(-)^{n} n q^{n^{2}}\left(t^{n}-t^{-n}\right)
$$

for the different values of $t=q z / \zeta$ which occur. Then

$$
f(z, \zeta) z^{\prime}=\left(\frac{t \vartheta_{*}^{\prime}(t)}{\vartheta_{*}(t)}+\frac{1}{2} \frac{\log |\zeta|}{\log q}\right) \frac{z^{\prime}}{z} .
$$

The conjugate function $v$ is given by the integral

$$
v=\frac{1}{2 \pi} \int_{C} \mu(z) d \tilde{f}(z, \zeta) z^{\prime} d \sigma,
$$

where

$$
\tilde{f}(z, \zeta) z^{\prime}=\left(\frac{-i t \vartheta_{*}^{\prime}(t)}{\vartheta_{*}(t)}+\frac{1}{2} \frac{\arg \zeta}{\log q}\right) \frac{z^{\prime}}{z}
$$

and 


$$
d \tilde{f}(z, z) z^{\prime} \sim \frac{1}{\sigma},
$$

with primes denoting differentiation with respect to $\sigma$. The integral (35) has to be taken to mean its Cauchy principle value if $\zeta \in C$. However, since

$$
\int_{C} d f(z, \zeta) z^{\prime} d \sigma=0,
$$

we may write (35) as

$$
v=\frac{1}{2 \pi} \int_{C}[\mu(z)-\mu(\zeta)] d f(z, \zeta) z^{\prime} \cdot d \sigma,
$$

with

$$
\left.[\mu(z)-\mu(\zeta)] d f(z, \zeta) z^{\prime}\right|_{z=\zeta}=\frac{\partial \mu}{\partial \zeta} .
$$

6. An application to conformal mapping. In order to map the doubly connected region $\Omega$ onto the annulus $r \leq|w| \leq 1$, it is only necessary to find, say using the method of the preceding section, that analytic function $u+i v$ in $\Omega$ for which $u=0$ on the outer boundary of $\Omega$ and $u=1$ on the inner contour. For if $\omega$ is the period of $v$, then

$$
w=e^{(2 \pi / \omega)(u+i v)}
$$

maps $\Omega$ onto the annulus, and $v / \omega$ gives the angular correspondence on the boundaries.

\section{REFERENCES}

1. L. Ahlfors, Remarks on the Neumann-Poincaré integral equation, this issue p.273282 .

2. S. Bergman and M. Schiffer, Kernel functions and conformal mapping, Compositio Math. 8 (1951), 205-249.

3. G. Birkhoff, D. M. Young and E. H. Zarantonello, Effective conformal transformation of smooth, simply connected domains, Proc. Nat. Acad. Sci., 37 (1951).

4. - Numerical methods in conformal mapping. To appear in NBS Applied Math. Series 18.

5. H. Poincaré, La methode de Neumann et le probleme de Dirichlet. Acta Math. 20 (1897), 59-142.

6. E. T. Whittaker and G. N. Watson, Modern analysis, Cambridge, England, 1940. 


\title{
PACIFIC JOURNAL OF MATHEMATICS
}

\section{EDITORS}

\section{R. M. RobINSON \\ University of California \\ Berkeley 4, California}

\author{
*R. P. Dilwor TH \\ California Institute of Technology \\ Pasadena 4, California
}

\author{
E. F. BECKENBACH, Managing Editor \\ University of California \\ Los Angeles 24, California
}

${ }^{*}$ During the absence of Herbert Busemann in 1952.

\section{ASSOCIATE EDITORS}

$\begin{array}{llll}\text { R. P. DILWORTH } & \text { P. R. HALMOS } & \text { B } \emptyset \text { RGE JESSEN } & \text { J. J. STOKER } \\ \text { HERBERT FEDERER } & \text { HEINZ HOPF } & \text { PAUL LÉVY } & \text { E. G. STRAUS } \\ \text { MARSHALL HALL } & \text { R. D. JAMES } & \text { GEORGE PÓLYA } & \text { KÖSAKU YOSIDA }\end{array}$

\section{SPONSORS}

UNIVERSITY OF BRITISH COLUMBIA

CALIFORNIA INSTITUTE OF TECHNOLOGY

U NIVERSITY OF CALIFORNIA, BERKELEY

UNIVERSITY OF CALIFORNIA, DAVIS

UNIVERSITY OF CALIFORNIA, LOS ANGELES

UNIVERSITY OF CALIFORNIA, SANTA BARBARA

OREGON STATE COLLEGE

UNIVERSITY OF OREGON

\author{
UNIVERSITY OF SOUTHERN CALIFORNIA \\ STANFORD UNIVERSITY \\ WASHINGTON STATE COLLEGE \\ UNIVERSITY OF WASHINGTON \\ AMERICAN MATHEMATICAL SOCIETY \\ NATIONAL BUREAU OF STANDARDS, \\ INSTITUTE FOR NUMERICAL ANALYSIS
}

Mathematical papers intended for publication in the Pacific Journal of Miathematics should be typewritten (double spaced), and the author should keep a complete copy. Manuscripts may be sent to any of the editors. All other communications to the editors should be addressed to the managing editor, E. F. Beckenbach, at the address given above.

Authors are entitled to receive 100 free reprints of their published papers and may obtain additional copies at cost.

The Pacific Journal of Mathematics is published quarterly, in March, June, September, and December, by the University of California, Berkeley 4, California. The price per volume (4 numbers) is $\$ 8.00$; single issues, $\$ 2.50$. Special price to individual faculty members of supporting institutions and to individual members of the American Mathematical Society: $\$ 4.00$ per volume; single issues, $\$ 1.25$.

Subscriptions, orders for back numbers, and changes of address should be sent to the publishers, University of California Press, Berkeley 4, California.

Printed at Ann Arbor, Michigan. Entered as second class matter at the Post Office, Berkeley, California.

\section{UNIVERSITY OF CALIFORNIA PRESS • BERKELEY AND LOS ANGELES}




\section{Pacific Journal of Mathematics}

\section{Vol. 2, No. $3 \quad$ March, 1952}

Lars V. Ahlfors, Remarks on the Neumann-Poincaré integral equation .... 271

Leonard P. Burton, Oscillation theorems for the solutions of linear, nonhomogeneous, second-order differential systems ............ 281

Paul Civin, Multiplicative closure and the Walsh functions . . . . . . . . . . . 291

James Michael Gardner Fell and Alfred Tarski, On algebras whose factor algebras are Boolean .................................. 297

Paul Joseph Kelly and Lowell J. Paige, Symmetric perpendicularity in Hilbert geometries .................................. 319

G. Kurepa, On a characteristic property of finite sets .............. 323

Joseph Lehner, A diophantine property of the Fuchsian groups ......... 327

Donald Alan Norton, Groups of orthogonal row-latin squares ........... 335

R. S. Phillips, On the generation of semigroups of linear operators ....... 343

G. Piranian, Uniformly accessible Jordan curves through large sets of relative harmonic measure zero ........................ 371

C. T. Rajagopal, Note on some Tauberian theorems of $O . S z \tilde{A} ; s z \ldots \ldots \ldots 377$

Halsey Lawrence Royden, Jr., A modification of the Neumann-Poincaré method for multiply connected regions .................... 385

George H. Seifert, A third order irregular boundary value problem and the associated series ...................................... 395

Herbert E. Vaughan, Well-ordered subsets and maximal members of ordered sets....

Hans F. Weinberger, An optimum problem in the Weinstein method for eigenvalues.

Shigeki Yano, Note on Fourier analysis. XXXI. Cesàro summability of Fourier series. 